UDC 517.938.5

\author{
E. Mukhamadiev, A. N. Naimov
}

\title{
ON THE HOMOTOPY CLASSIFICATION OF POSITIVELY HOMOGENEOUS FUNCTIONS OF THREE VARIABLES
}

\begin{abstract}
In this paper, we study the problem of homotopy classification of the set $\mathcal{F}$ of positively homogeneous smooth functions in three variables whose gradients do not vanish at nonzero points. This problem is of interest in the study of periodic and bounded solutions of systems of ordinary differential equations with the main positive homogeneous nonlinearity. The subset $\mathcal{F}_{0} \subset \mathcal{F}$ is presented and for any function $g(x) \in \mathcal{F}_{0}$, a formula for calculating the rotation $\gamma(\nabla g)$ of its gradient $\nabla g(x)$ on the boundary of the unit ball $|x|<1$ is derived. It is proved that any function from $\mathcal{F}$ is homotopic to some function from $\mathcal{F}_{0}$.
\end{abstract}

Key words: positively homogeneous function, homotopy, homotopy classification, vector field rotation

2010 Mathematical Subject Classification: 26A21, 54C50

1. Introduction. The article investigates the problem of homotopy classification of the set $\mathcal{F}$ of functions $f(x)$ satisfying the conditions

a) $f \in C^{1}\left(\mathbb{R}^{3} \backslash\{0\} ; \mathbb{R}^{1}\right)$;

b) $f(\lambda x) \equiv \lambda^{m} f(x) \forall \lambda>0$, where $m=m(f) \neq 0$;

c) $\nabla f(x) \neq 0 \forall x \neq 0$, where $\nabla f(x)=\left(\frac{\partial f}{\partial x_{1}}, \frac{\partial f}{\partial x_{2}}, \frac{\partial f}{\partial x_{3}}\right)$.

This problem is important when studying periodic and bounded solutions of systems of ordinary differential equations with the main positive homogeneous nonlinearity [3], [4], [5].

The problem of homotopy classification of the set $\mathcal{F}$ consists of describing homotopy classes of functions. Two functions $f_{1}, f_{2} \in \mathcal{F}$ are called homotopic if there exists a family of functions $\widetilde{f}(\cdot, t) \in \mathcal{F}, t \in[0,1]$, continuously depending on $t$ and such that $\widetilde{f}(\cdot, 0)=f_{1}, \widetilde{f}(\cdot, 1)=f_{2}$.

(c) Petrozavodsk State University, 2021 
It follows from the results of [4] that the functions $f_{1}, f_{2} \in \mathcal{F}$ are homotopic if and only if the corresponding sets are similar:

$$
\Omega_{-}\left(f_{i}\right)=\left\{x \in \mathbb{R}^{3}:|x|=1, f_{i}(x)<0\right\}, \quad i=1,2 .
$$

The similarity of the sets $\Omega_{-}\left(f_{i}\right), i=1,2$ is defined by means of the existence of a family of diffeomorphisms $G(\cdot, \mu): S^{2} \mapsto S^{2}, \mu \in[0,1]$, where $S^{2}=\left\{x \in \mathbb{R}^{3}:|x|=1\right\}$, which depends continuously on $\mu$ and satisfies the conditions $G(x, 0) \equiv x, G\left(\Omega_{-}\left(f_{1}\right), 1\right)=\Omega_{-}\left(f_{2}\right)$. Establishing similarity of sets $\Omega_{-}\left(f_{i}\right), i=1,2$ is a topological problem.

In this work, the following results are obtained:

1. The subset $\mathcal{F}_{0} \subset \mathcal{F}$ is presented: it consists of functions of the form

$$
\begin{gathered}
g(x)=|x|^{\nu}\left(\left\langle c_{1}, x\right\rangle-d_{1}|x|\right) \cdot \ldots \cdot\left(\left\langle c_{p}, x\right\rangle-d_{p}|x|\right), \\
\nu, d_{i} \in \mathbb{R}^{1}, c_{i} \in \mathbb{R}^{3}, i=\overline{1, p},|x|^{2}=\langle x, x\rangle,\langle x, y\rangle=x_{1} y_{1}+x_{2} y_{2}+x_{3} y_{3} .
\end{gathered}
$$

2. A formula for calculating the rotation $\gamma(\nabla g)$ of the gradient $\nabla g$ of the function $g \in \mathcal{F}_{0}$ on $S^{2}$ is derived.

3. It is proved that any function from $\mathcal{F}$ is homotopic to some function from $\mathcal{F}_{0}$.

Thus, functions of the form (1) are representatives of all classes of homotopic functions from $\mathcal{F}$. The results obtained in this work can subsequently be extended to functions of four or more variables.

2. Main results. Let $g(x)$ be a function of the form (1). Let us find out, under what conditions $g(x)$ belongs to the set $\mathcal{F}$. The following theorem is true:

Theorem 1. The function $g(x)$ defined by the formula (1) belongs to $\mathcal{F}$ if and only if the following conditions are satisfied:

$A_{1}:(\nu+p) \neq 0$

$A_{2}:\left|c_{i}\right| \neq\left|d_{i}\right|, i=1, \ldots, p$;

$A_{3}$ : for any $i, j \in\{1, \ldots, p\}, i \neq j$, the implication is correct:

$$
\left(\left|c_{i}\right|>\left|d_{i}\right|,\left|c_{j}\right|>\left|d_{j}\right|\right) \Longrightarrow\left|d_{i} c_{j}-d_{j} c_{i}\right|>\left(\left|c_{i}\right|^{2}\left|c_{j}\right|^{2}-\left\langle c_{i}, c_{j}\right\rangle^{2}\right)^{1 / 2} .
$$

If the conditions $A_{1}-A_{3}$ are satisfied, then for the rotation $\gamma(\nabla g)$ of the vector field $\nabla g$ on $S^{2}$ the following formula holds:

$$
\gamma(\nabla g)=p_{+}(g)-p_{-}(g)
$$


where $p_{ \pm}(g)$ is the number of connected components of the set

$$
\Omega_{ \pm}(g)=\left\{x \in S^{2}: \pm g(x)>0\right\} .
$$

Denote by $\mathcal{F}_{0}$ the set of functions of the form (1) under the conditions $A_{1}-A_{3}$.

By virtue of the results of [4], the homotopy classification of the set $\mathcal{F}$ is equivalent to describing the structure of the sets $\Omega_{-}(f), f \in \mathcal{F}$ up to similarity. The structures of these sets are completely determined by functions from $\mathcal{F}_{0}$. Namely, the following theorem holds:

Theorem 2. Any function $f \in \mathcal{F}$ is homotopic to some $g \in \mathcal{F}_{0}$.

The values of $\gamma(\nabla f), p_{ \pm}(f)$ do not change under homotopies of the function $f \in \mathcal{F}$. Therefore Theorems 1 and 2 imply the following

Corollary. For $f \in \mathcal{F}$, the formula holds: $\gamma(\nabla f)=p_{+}(f)-p_{-}(f)$, where $p_{ \pm}(f)$ is the number of connected components of the set $\Omega_{ \pm}(f)$.

3. Let us present the proofs of Theorems 1 and 2. First, let us check the following lemma:

Lemma 1. For two positively homogeneous functions $f_{i}(\lambda x) \equiv \lambda^{m_{i}} f_{i}(x)$, $f_{i} \in C^{1}\left(\mathbb{R}^{3} \backslash\{0\} ; \mathbb{R}^{1}\right), i=1,2$, where the numbers $m_{1}, m_{2}, m_{1}+m_{2}$ are nonzero, the product $f_{1} f_{2}$ belongs to the set $\mathcal{F}$ if and only if $f_{1}, f_{2} \in \mathcal{F}$ and $\left|f_{1}(x)\right|+\left|f_{2}(x)\right|>0 \forall x \neq 0$.

Proof. For any positively homogeneous function $f \in C^{1}\left(\mathbb{R}^{3} \backslash\{0\} ; \mathbb{R}^{1}\right)$, $f(\lambda x) \equiv \lambda^{m} f(x)$ the Euler formula holds: $m f(x)=\langle\nabla f(x), x\rangle$ for $x \neq 0$. Therefore, for such a function with $m \neq 0$, the inclusion $f \in \mathcal{F}$ is equivalent to the condition $|f(x)|+|\nabla f(x)|>0 \forall x \neq 0$.

Let $f_{1} f_{2} \in \mathcal{F}$. Then, for any $x \neq 0$ we have:

$$
\left|f_{1}(x) f_{2}(x)\right|+\left|f_{2}(x) \nabla f_{1}(x)+f_{1}(x) \nabla f_{2}(x)\right|>0 .
$$

Hence, the following inequalities hold for any $x \neq 0$ :

$$
\left|f_{1}(x)\right|+\left|f_{2}(x)\right|>0, \quad\left|f_{i}(x)\right|+\left|\nabla f_{i}(x)\right|>0, \quad i=1,2 .
$$

Consequently, $f_{1}, f_{2} \in \mathcal{F}$.

Conversely, if $f_{1}, f_{2} \in \mathcal{F}$ and $\left|f_{1}(x)\right|+\left|f_{2}(x)\right|>0 \forall x \neq 0$, then for any $x \neq 0$ the inequalities (4) hold. Then, for any $x \neq 0$ the following cases are possible: 
1) $f_{1}(x) f_{2}(x) \neq 0$

2) $f_{1}(x)=0, f_{2}(x) \neq 0$ and $\nabla f_{1}(x) \neq 0, f_{2}(x) \nabla f_{1}(x) \neq 0$;

3) $f_{1}(x) \neq 0, f_{2}(x)=0$ and $\nabla f_{2}(x) \neq 0, f_{1}(x) \nabla f_{2}(x) \neq 0$.

In all cases, the inequality $(3)$ is true. Hence, $f_{1} f_{2} \in \mathcal{F}$.

Lemma 1 is proved.

Proof of Theorem 1. Lemma 1 implies that the function $g(x)$, defined by the formula (1), belongs to the set $\mathcal{F}$ if and only if the conditions $A_{1}$ and

$A_{2}^{\prime}:\left\langle c_{i}, x\right\rangle-d_{i}|x| \in \mathcal{F}, i=1, \ldots, p ;$

$A_{3}^{\prime}$ : the following inequality holds for any $i, j \in\{1, \ldots, p\}, i \neq j, x \neq 0$ :

$$
\left|\left\langle c_{i}, x\right\rangle-d_{i}\right| x||+\left|\left\langle c_{j}, x\right\rangle-d_{j}\right| x||>0
$$

are fulfilled.

It is easy to check that the condition $A_{2}^{\prime}$ is equivalent to the condition $A_{2}$. Let us show that under the conditions $A_{1}, A_{2}$, the conditions $A_{3}^{\prime}$ and $A_{3}$ are equivalent. For this, we clarify the conditions for the unsolvability of the system of equations

$$
\left\langle c_{i}, x\right\rangle=d_{i}, \quad\left\langle c_{j}, x\right\rangle=d_{j}, \quad|x|=1
$$

for fixed $i, j$ and assuming $\left|c_{i}\right| \neq\left|d_{i}\right|,\left|c_{j}\right| \neq\left|d_{j}\right|$. Obviously, the system of equations (5) is not solvable if $\left|c_{i}\right|<\left|d_{i}\right|$ or $\left|c_{j}\right|<\left|d_{j}\right|$.

Consider the case when $\left|c_{i}\right|>\left|d_{i}\right|,\left|c_{j}\right|>\left|d_{j}\right|$. If the vectors $c_{i}$ and $c_{j}$ are collinear $c_{i}=\lambda c_{j}$, then the system of equations (5) is not solvable if and only if $d_{i} \neq \lambda d_{j}$. In this case, the conditions $A_{3}^{\prime}$ and $A_{3}$ are equivalent.

Let the vectors $c_{i}$ and $c_{j}$ are not collinear. Then any vector $x \in \mathbb{R}^{3}$ can be represented as $x=\alpha c_{i}+\beta c_{j}+h$, where $\left\langle c_{i}, h\right\rangle=0,\left\langle c_{j}, h\right\rangle=0$, and the system of equations (5) can be written as follows:

$$
\left\{\begin{array}{l}
\left|c_{i}\right|^{2} \alpha+\left\langle c_{i}, c_{j}\right\rangle \beta=d_{i}, \\
\left\langle c_{i}, c_{j}\right\rangle \alpha+\left|c_{j}\right|^{2} \beta=d_{j}, \\
\left|\alpha c_{i}+\beta c_{j}\right|^{2}+|h|^{2}=1 .
\end{array}\right.
$$

From the first two equations, one can find $\alpha$ and $\beta$ :

$$
\alpha=\frac{d_{i}\left|c_{j}\right|^{2}-d_{j}\left\langle c_{i}, c_{j}\right\rangle}{\left|c_{i}\right|^{2}\left|c_{j}\right|^{2}-\left\langle c_{i}, c_{j}\right\rangle^{2}}, \quad \beta=\frac{d_{j}\left|c_{i}\right|^{2}-d_{i}\left\langle c_{i}, c_{j}\right\rangle}{\left|c_{i}\right|^{2}\left|c_{j}\right|^{2}-\left\langle c_{i}, c_{j}\right\rangle^{2}} .
$$


Therefore, the system of equations (6) is not solvable only in the case when

$$
\left|\alpha c_{i}+\beta c_{j}\right|>1
$$

Substituting the values $\alpha$ and $\beta$ into this inequality, we get:

$$
\left|d_{i} c_{j}-d_{j} c_{i}\right|>\left(\left|c_{i}\right|^{2}\left|c_{j}\right|^{2}-\left\langle c_{i}, c_{j}\right\rangle^{2}\right)^{1 / 2}
$$

The equivalence of the conditions $A_{3}^{\prime}$ and $A_{3}$ is proved. This proves that the function $g(x)$, defined by the formula (1), belongs to the set $\mathcal{F}$ if and only if the conditions $A_{1}-A_{3}$ are satisfied.

Now prove the formula (2), assuming that the conditions $A_{1}-A_{3}$ are satisfied. For the rotation $\gamma(\nabla g)$ of the vector field $\nabla g$ on $S^{2}$, according to the results of [3, p. 597], the following formula is valid:

$$
\gamma(\nabla g)=1-\chi\left(\bar{\Omega}_{-}(g)\right)
$$

where $\chi\left(\bar{\Omega}_{-}(g)\right)$ is the Euler characteristic of the closure $\bar{\Omega}_{-}(g)$ of the set $\Omega_{-}(g)$. To find $\chi\left(\bar{\Omega}_{-}(g)\right)$, we use the following well-known properties the Euler characteristic [2]:

$1^{\circ}$. If $A, B, A \cup B, A \cap B$ are polyhedra, then

$$
\chi(A \cup B)=\chi(A)+\chi(B)-\chi(A \cap B) .
$$

$2^{\circ}$. If the boundary $\partial A$ of the polyhedron $A \subset S^{2}$ consists of $k$ disjoint circles, then $\chi(A)=2-k$.

The set $\bar{\Omega}_{-}(g)$ has a smooth boundary, therefore it is a polyhedron. We represent it as a union of connected components

$$
\bar{\Omega}_{-}(g)=\bar{\Omega}_{-, 1} \cup \ldots \cup \bar{\Omega}_{-, p_{-}} .
$$

In accordance with the properties of $1^{\circ}$ and $2^{\circ}$, we have:

$$
\begin{gathered}
\chi\left(\bar{\Omega}_{-}(g)\right)=\chi\left(\bar{\Omega}_{-, 1}\right)+\ldots+\chi\left(\bar{\Omega}_{-, p_{-}}\right), \\
\chi\left(\bar{\Omega}_{-, i}\right)=2-k_{i}, \quad i=1, \ldots, p_{-},
\end{gathered}
$$

where $k_{i}$ is the number of connected components $\partial \bar{\Omega}_{-, i}$. We substitute these values into the formula (7):

$$
\gamma(\nabla g)=1-2 p_{-}+\left(k_{1}+\ldots+k_{p_{-}}\right) .
$$


Taking into account the easily verified equality $k_{1}+\ldots+k_{p_{-}}=p_{+}+p_{-}-1$, we get the formula (2).

Theorem 1 is proved.

Let $f \in \mathcal{F}$ has homogeneity of order $m$. Consider the set

$$
\Omega_{0}(f)=\left\{x \in S^{2}: f(x)=0\right\} .
$$

If $\Omega_{0}(f)$ is empty, then $f$ and $\operatorname{sign}(f)|x|^{m}$ are linear homotopic: $(1-t) f+t \operatorname{sign}(f)|x|^{m} \in \mathcal{F}$ for $t \in[0,1]$. In this case, Theorem 2 is true.

If $\Omega_{0}(f)$ is not empty, then it consists of a finite number of connected components:

$$
\Omega_{0}(f)=\Omega_{0,1}(f) \cup \ldots \cup \Omega_{0, p}(f) .
$$

Each connected component $\Omega_{0, i}(f)$ is a smooth line, diffeomorphic to a circle. If all the lines $\Omega_{0, i}(f), i=1, \ldots, p$ are circles, then the equalities $\Omega_{0, i}(f)=\Omega_{0}\left(g_{i}\right), i=1, \ldots, p$ hold for some $g_{i}(x)=\left\langle c_{i}, x\right\rangle-d_{i}|x| \in \mathcal{F}$, $i=1, \ldots, p$. It implies that $g=g_{1} \cdot \ldots \cdot g_{p} \in \mathcal{F}_{0}$ (due to Lemma 1) and $f$ is linearly homotopic to $g$. It remains to consider the case when not all lines $\Omega_{0, i}(f), i=1, \ldots, p$ are circles. Conceptually it is clear that they can be continuously transformed into the circles and thus define a homotopy of $f$ to some function $g=g_{1} \cdot \ldots \cdot g_{p} \in \mathcal{F}_{0}$. We will further implement this idea.

First, we prove a lemma on the representation of the function $f$.

Lemma 2. The function $f$ can be represented as

$$
f(x)=f_{1}(x) \cdot \ldots \cdot f_{p}(x)|x|^{m-p},
$$

where $f_{i} \in \mathcal{F}, m_{i}=m_{i}\left(f_{i}\right)=1, \Omega_{0}\left(f_{i}\right)=\Omega_{0, i}(f), i=1, \ldots, p$.

Proof. For $p=1$, the representation (8) is obvious, therefore, we consider $p>1$. Let us choose $\delta>0$ so small that the set

$$
\Omega_{2 \delta}(f)=\left\{x \in S^{2}:|f(x)|<2 \delta\right\}
$$

consists of exactly $p$ connected components:

$$
\Omega_{2 \delta}(f)=\Omega_{2 \delta, 1}(f) \cup \ldots \cup \Omega_{2 \delta, p}(f),
$$

where $\Omega_{0, i}(f) \subset \Omega_{2 \delta, i}(f)$. For every $i=1, \ldots, p-1$ we put

$$
f_{i}(x)=\left\{\begin{array}{cc}
\delta \Psi\left(\delta^{-1} f\left(\frac{x}{|x|}\right)\right)|x|, & \frac{x}{|x|} \in \Omega_{2 \delta, i}(f), \\
\pm 2 \delta|x|, & \frac{x}{|x|} \notin \Omega_{2 \delta, i}(f), \quad \pm f\left(\frac{x}{|x|}\right) \geqslant 2 \delta
\end{array}\right.
$$


where

$$
\Psi(t)=\left\{\begin{array}{cl}
t, & 0 \leqslant t \leqslant 1, \\
t+(2-t)(1-t)^{2}, & 1<t<2, \\
2, & t \geqslant 2,
\end{array}\right.
$$

and $\Psi(t)=-\Psi(-t)$ for $t<0$. It is easy to check that $f_{i} \in \mathcal{F}$, $\Omega_{0}\left(f_{i}\right)=\Omega_{0, i}(f), i=1, \ldots, p-1$. We continuously extend the function $f_{p}$, defined by the formula $f_{p}(x)=f(x)\left(f_{1}(x) \cdot \ldots \cdot f_{p-1}(x)\right)^{-1}$ for $x \in S^{2} \backslash\left(\Omega_{0,1}(f) \cup \ldots \cup \Omega_{0, p-1}(f)\right)$, to $S^{2}$ and to $\mathbb{R}^{3} \backslash\{0\}$ positively homogeneously with order 1 . Then $f_{p} \in \mathcal{F}, \Omega_{0}\left(f_{p}\right)=\Omega_{0, p}(f)$ and the representation (8) is true.

Lemma 2 is proved.

Proof of Theorem 2. The functions $f_{i}, i=1, \ldots, p$ are homotoped to some functions $g_{i}(x)=\left\langle c_{i}, x\right\rangle-d_{i}|x| \in \mathcal{F}, i=1, \ldots, p$ so that the sets of zeros do not intersect under the homotopy. Then, according to Lemma 1, the function $f$ is homotopic to the function $g=g_{1} \cdot \ldots \cdot g_{p}$.

Subsequently, without loss of generality, we can assume that $f_{i} \in C^{\infty}\left(\mathbb{R}^{3} \backslash\{0\} ; \mathbb{R}^{1}\right), i=1, \ldots, p$, since any function from $\mathcal{F}$ is linearly homotopic to some function from $\mathcal{F} \cap C^{\infty}\left(\mathbb{R}^{3} \backslash\{0\} ; \mathbb{R}^{1}\right)$ (see, for example, [4, p. 122], [5, p. 164]). In this case, the lines $\Omega_{0}\left(f_{i}\right), i=1, \ldots, p$, are infinitely smooth. We apply two types of transformations to these lines: $T_{\varepsilon}(\Gamma, C)$ transforms a closed smooth line $\Gamma$ to a circle $C, T_{a, h, \varepsilon}(C)$ is the parallel translation of the circle $C$ in the direction of the unit vector $a$ by length $h$.

The transformation $T_{\varepsilon}(\Gamma, C)$ is applied under the following conditions: $\Gamma, C \subset S^{2}$, the closed line $\Gamma$ is sufficiently smooth and diffeomorphic to a circle, the circle $C$ lies inside the domain bounded by $\Gamma$. The transformation $T_{\varepsilon}(\Gamma, C)$ is a family of diffeomorphisms $G_{\varepsilon}(\cdot, \mu): S^{2} \mapsto S^{2}, \mu \in[0,1]$, continuously depending on $\mu$ and having the following properties:

$1^{\circ} . G_{\varepsilon}(x, \mu)=x$, if $\mu=0, x \in S^{2}$ or $\mu \in[0,1], x \notin D_{\varepsilon}(\Gamma, C)$, where $D_{\varepsilon}(\Gamma, C)$ is an $\varepsilon$-neighborhood of the domain $D(\Gamma, C)$, bounded by the lines $\Gamma$ and $C$.

$$
2^{\circ} . G_{\varepsilon}(\Gamma, 1)=C \text {. }
$$

The transformation $T_{a, h, \varepsilon}(C)$ is applied to the circle $C \subset S^{2}$ and is a family of diffeomorphisms $G_{a, h, \varepsilon}(\cdot, \mu): S^{2} \mapsto S^{2}, \mu \in[0,1]$, continuously depending on $\mu$ and having the following properties:

$3^{\circ} . G_{a, h, \varepsilon}(x, \mu)=x$, if $\mu=0, x \in S^{2}$ or $\mu \in[0,1], x \notin K_{h+\varepsilon}$, where $K_{h+\varepsilon}$ is a disk, whose center coincides with the center of the circle $C$ and has radius $h+\varepsilon$ longer than the radius of $C$. 
$4^{\circ} . G_{a, h, \varepsilon}(C, 1)=C^{\prime}$, where $C^{\prime}$ is the circle obtained from $C$ by parallel translation in the direction of the vector $a$ by length $h$.

Homotopies of the functions $f_{i}, i=1, \ldots, p$ are implemented according to the following algorithm:

1. We transform all lines $\Omega_{0}\left(f_{i}\right)$, the interior or exterior of which does not contain other lines through the appropriate transformations $T_{\varepsilon_{i}}\left(\Omega_{0}\left(f_{i}\right), C_{i}\right)$, into the circles $C_{i}$ of sufficiently small radii. These transformations generate homotopies of the corresponding functions $f_{i}$ to some functions $g_{i}$ from $\mathcal{F}_{0}$.

2. We transfer and concentrate each group of circles obtained at the first step and not separated from each other by other lines in pairs, around certain points by repeatedly applying transformations of the second type $T_{a, h, \varepsilon}(C)$. These transformations generate homotopies of the functions $g_{i} \in \mathcal{F}_{0}$ obtained at the first step.

3 . We repeat the previous steps until all lines $\Omega_{0}\left(f_{i}\right)$ are converted into a circle. If necessary, we homotop certain circles to circles of smaller radii. As a result, the function $f=f_{1} \cdot \ldots \cdot f_{p}$ is homotoping to the function $g=g_{1} \cdot \ldots \cdot g_{p} \in \mathcal{F}_{0}$.

To justify the above algorithm, it is necessary to show the possibility of constructing the transformations $T_{\varepsilon}(\Gamma, C)$ and $T_{a, h, \varepsilon}(C)$. It is sufficient to construct these transformations on the plane $\mathbb{R}^{2}$, then they can be transferred to the sphere $S^{2}$ by a stereographic mapping $S^{2}$ with a punctured point on $\mathbb{R}^{2}$.

Let $\Gamma$ be an infinitely smooth and closed line on the plane $\mathbb{R}^{2}$, diffeomorphic to a circle and being the boundary of a bounded simply connected domain $D$. Fix the point $z_{0} \in D$ and choose $r_{0}>0$ so that the disk $K_{r_{0}}\left(z_{0}\right)=\left\{z \in \mathbb{R}^{2}:\left|z-z_{0}\right|<r_{0}\right\}$ with boundary $C_{r_{0}}\left(z_{0}\right)$ is inside the domain $D$.

Let $D\left(\Gamma, C_{r_{0}}\left(z_{0}\right)\right)$ be the domain bounded by the lines $\Gamma$ and $C_{r_{0}}\left(z_{0}\right)$. Supplement this domain with the set $\prod_{\varepsilon_{0}}\left(\Gamma, C_{r_{0}}\left(z_{0}\right)\right)$ of points $\eta+l \cdot n(\eta)$, where $\eta \in \Gamma \cup C_{r_{0}}\left(z_{0}\right), l \in\left(0, \varepsilon_{0}\right), n(\eta)$ is a unit outer normal vector at the point $\eta$. Here the number $\varepsilon_{0} \in\left(0, r_{0}\right)$ is so small that in any point $z \in \bar{\Pi}_{\varepsilon_{0}}\left(\Gamma, C_{r_{0}}\left(z_{0}\right)\right)$ has a unit decomposition $z=\eta(z)+l(z) n(\eta(z))$, where $\eta(z) \in \Gamma \cup C_{r_{0}}\left(z_{0}\right), l(z) \in\left[0, \varepsilon_{0}\right]$. The functions $\eta(z)$ and $l(z)$ are defined on $\bar{\Pi}_{\varepsilon_{0}}\left(\Gamma, C_{r_{0}}\left(z_{0}\right)\right)$ and are smooth due to the fact that the line $\Gamma$ is assumed to be infinitely smooth. For any $\varepsilon \in\left(0, \varepsilon_{0}\right)$, similarly, we define $\Pi_{\varepsilon / 2}\left(\Gamma, C_{\varepsilon}\left(z_{0}\right)\right)$ and denote $D_{\varepsilon}\left(\Gamma, C_{\varepsilon}\left(z_{0}\right)\right)=\bar{D}\left(\Gamma, C_{\varepsilon}\left(z_{0}\right)\right) \cup \Pi_{\varepsilon / 2}\left(\Gamma, C_{\varepsilon}\left(z_{0}\right)\right)$.

The next lemma implies the possibility of constructing the above trans- 
formation $T_{\varepsilon}(\Gamma, C)$ for circles $C$ of small radius.

Lemma 3. There is $\varepsilon_{1} \in\left(0, \varepsilon_{0}\right)$, such that for $\varepsilon \in\left(0, \varepsilon_{1}\right)$ one can construct a family of diffeomorphisms $G_{\varepsilon}(\cdot, \mu): \mathbb{R}^{2} \mapsto \mathbb{R}^{2}, \mu \in[0,1]$, continuously depending on $\mu$ and satisfying the conditions

1) $G_{\varepsilon}(z, \mu)=z$, if $\mu=0, z \in \mathbb{R}^{2}$ or $\mu \in[0,1], z \notin D_{\varepsilon}\left(\Gamma, C_{\varepsilon}\left(z_{0}\right)\right)$;

2) $G_{\varepsilon}(\Gamma, 1)=C_{\varepsilon}\left(z_{0}\right)$.

Proof. We construct the family of diffeomorphisms $G_{\varepsilon}(\cdot, \mu): \mathbb{R}^{2} \mapsto \mathbb{R}^{2}$, $\mu \in[0,1]$ along the trajectories of a specially constructed two-dimensional autonomous system. To construct an autonomous system, we use the Riemann theorem on the conformal mapping of a two-dimensional simply connected domain onto a circle and the Kellogg theorem on the smooth extension of the conformal mapping to the smooth boundary of the domain [1, Ch. 10, §1]. According to the Riemann theorem, there is a continuous mapping $\Phi: \bar{D} \mapsto \bar{K}_{r_{0}}\left(z_{0}\right)$, conformal inside $D$ and satisfying the conditions $\Phi(\Gamma)=C_{r_{0}}\left(z_{0}\right), \Phi\left(z_{0}\right)=z_{0}$. This mapping is continuously differentiable on $\bar{D}$ according to the Kellogg theorem.

Consider the function $u(z)=\left|\Phi(z)-z_{0}\right|^{2}, z \in \bar{D}$. One can check directly that $u \in C^{1}\left(\bar{D} ; \mathbb{R}^{1}\right), \nabla u(z) \neq 0 \forall z \in \bar{D} \backslash\left\{z_{0}\right\}, \nabla u(\eta) \| n(\eta) \forall \eta \in \Gamma$, where $n(\eta)$ is a unit outer normal vector at the point $\eta \in \Gamma$. Taking into account that $z_{0}$ is a minimum point of the function $u$ and the mapping $\Phi$ is conformal in a neighborhood of the point $z_{0}$, we choose $\varepsilon_{1} \in\left(0, \varepsilon_{0}\right)$ so that for $\left|z-z_{0}\right| \leqslant \varepsilon_{1}$ the inequality $\left\langle\nabla u(z), z-z_{0}\right\rangle \geqslant \alpha\left|z-z_{0}\right|^{2}$ holds; here $\alpha>0$ and does not depend on $z$. For any $\varepsilon \in\left(0, \varepsilon_{1}\right)$, we define

$$
F_{\varepsilon}(z)=\left\{\begin{array}{cl}
\nabla u(z), & z \in \bar{D}\left(\Gamma, C_{\varepsilon}\left(z_{0}\right)\right), \\
\left(1-2 \varepsilon^{-1} l(z)\right) \nabla u(\eta(z)), & z \in \Pi_{\varepsilon / 2}\left(\Gamma, C_{\varepsilon}\left(z_{0}\right)\right) \backslash \bar{D}, \\
\left(1-2 \varepsilon^{-1} l(z)\right) \nabla u(z), & z \in \Pi_{\varepsilon / 2}\left(\Gamma, C_{\varepsilon}\left(z_{0}\right)\right) \cap K_{\varepsilon}\left(z_{0}\right), \\
0, & z \notin D_{\varepsilon}\left(\Gamma, C_{\varepsilon}\left(z_{0}\right)\right) .
\end{array}\right.
$$

Consider the following autonomous system:

$$
z^{\prime}(t)=F_{\varepsilon}(z(t)), \quad z(t) \in \mathbb{R}^{2} .
$$

For any point $\xi \in \mathbb{R}^{2}$, there is a unique solution $Z_{\varepsilon}(t, \xi)$ of the autonomous system, defined for all $t \in(-\infty,+\infty)$ and satisfying the initial condition $Z_{\varepsilon}(0, \xi)=\xi$. Any non-stationary solution $Z_{\varepsilon}(t, \xi)$ has the following properties:

1) the solution trajectory is located inside the domain $D_{\varepsilon}\left(\Gamma, C_{\varepsilon}\left(z_{0}\right)\right)$; 
2) the following limits exist: $Z_{\varepsilon}(t, \xi) \rightarrow P_{\varepsilon}^{-}(\xi)$ as $t \rightarrow-\infty$ and $Z_{\varepsilon}(t, \xi) \rightarrow P_{\varepsilon}^{+}(\xi)$ as $t \rightarrow+\infty$, where $P_{\varepsilon}^{-}(\xi)$ and $P_{\varepsilon}^{+}(\xi)$ are boundary points of the inner and outer boundaries of the domain $D_{\varepsilon}\left(\Gamma, C_{\varepsilon}\left(z_{0}\right)\right)$;

$3)$ there are unique moments $\tau_{C_{\varepsilon}\left(z_{0}\right)}(\xi)$ and $\tau_{\Gamma}(\xi)$ at which the inclusions $Z_{\varepsilon}\left(\tau_{C_{\varepsilon}\left(z_{0}\right)}(\xi), \xi\right) \in C_{\varepsilon}\left(z_{0}\right)$ and $Z_{\varepsilon}\left(\tau_{\Gamma}(\xi), \xi\right) \in \Gamma$ take place.

The latter follows from property 2$)$ and the above mentioned properties of the gradient of the function $u$ :

$$
\begin{gathered}
\nabla u(\eta) \| n(\eta) \quad \forall \eta \in \Gamma \\
\left\langle\nabla u(z), z-z_{0}\right\rangle \geqslant \alpha\left|z-z_{0}\right|^{2} \quad \text { for } \quad\left|z-z_{0}\right| \leqslant \varepsilon_{1} .
\end{gathered}
$$

The functions $\tau_{C_{\varepsilon}\left(z_{0}\right)}(\xi)$ and $\tau_{\Gamma}(\xi)$ are defined on the domain $D_{\varepsilon}\left(\Gamma, C_{\varepsilon}\left(z_{0}\right)\right)$ and are continuously differentiable by the implicit function theorem. The limits of these functions when $\xi$ approaches a point of the inner boundary are equal to $+\infty$, when $\xi$ approaches a point of the outer boundary they are equal to $-\infty$, and the difference $\left(\tau_{C_{\varepsilon}\left(z_{0}\right)}(\xi)-\tau_{\Gamma}(\xi)\right)$ is bounded.

We construct the family of diffeomorphisms $G_{\varepsilon}(\cdot, \mu): \mathbb{R}^{2} \mapsto \mathbb{R}^{2}$, $\mu \in[0,1]$ by the following formula:

$$
G_{\varepsilon}(\xi, \mu)=\left\{\begin{array}{cl}
\left.Z_{\varepsilon}\left(\lambda\left[\tau_{C_{\varepsilon}\left(z_{0}\right)}(\xi)-\tau_{\Gamma}(\xi)\right)\right], \xi\right), & \xi \in D_{\varepsilon}\left(\Gamma, C_{\varepsilon}\left(z_{0}\right)\right), \\
\xi, & \xi \notin D_{\varepsilon}\left(\Gamma, C_{\varepsilon}\left(z_{0}\right)\right) .
\end{array}\right.
$$

It can be verified directly that this family of diffeomorphisms satisfies the conditions 1) and 2) of the Lemma 3.

Lemma 3 is proved.

Now let us explain the possibility of constructing a transformation $T_{a, h, \varepsilon}(C)$. Let $C_{r}\left(z_{0}\right)$ be a circle of radius $r$ centered at $z_{0}$ on the plane $\mathbb{R}^{2}$. Let us set a unit vector $a \in \mathbb{R}^{2}$ and positive numbers $h, \varepsilon$. Consider the following autonomous system

$$
z^{\prime}(t)=\varphi_{h, \varepsilon}\left(\left|z(t)-z_{0}\right|\right) h a, \quad z(t) \in \mathbb{R}^{2}
$$

where

$$
\varphi_{h, \varepsilon}(\rho)=\left\{\begin{array}{cc}
1, & \rho \leqslant r+h, \\
0, & \rho \geqslant r+h+\varepsilon, \\
\frac{2}{\varepsilon^{3}}(r+h+\varepsilon-\rho)^{2}\left(\rho-r-h+\frac{\varepsilon}{2}\right), & r+h<\rho<r+h+\varepsilon .
\end{array}\right.
$$


We denote by $Z_{a, h, \varepsilon}(t, \xi)$ the solution of this autonomous system, satisfying the initial condition $Z_{a, h, \varepsilon}(0, \xi)=\xi$. If $\left|\xi-z_{0}\right| \leqslant r$, then

$$
\left|Z_{a, h, \varepsilon}(t, \xi)-z_{0}\right| \leqslant r+h \quad \text { for } \quad t \in[0,1]
$$

This implies that $Z_{a, h, \varepsilon}(t, \xi)=\xi+h t a$ for $\left|\xi-z_{0}\right| \leqslant r, t \in[0,1]$, in particular, $\left|Z_{a, h, \varepsilon}(1, \xi)-\left(z_{0}+h a\right)\right|=r$ for $\left|\xi-z_{0}\right|=r$.

Define a family of diffeomorphisms $G_{a, h, \varepsilon}(\xi, \mu)=Z_{a, h, \varepsilon}(\mu, \xi), \xi \in \mathbb{R}^{2}$, $\mu \in[0,1]$. It is easy to check that $G_{a, h, \varepsilon}(\xi, \mu)=\xi$ for $\left|\xi-z_{0}\right| \geqslant r+h+\varepsilon$ and $G_{a, h, \varepsilon}\left(C_{r}\left(z_{0}\right), 1\right)=C_{r}\left(z_{0}+h a\right)$. This family of diffeomorphisms defines the transformation $T_{a, h, \varepsilon}(C)$.

Theorem 2 is proved.

\section{References}

[1] Goluzin G. M. Geometric theory of functions of a complex variable. Moscow, Nauka, 1966 (in Russian). Translations of Mathematical Monographs, vol. 26, American Mathematical Society, Providence, R.I., 1969, MR 0247039.

[2] Borisovich Yu. G., Bliznyakov N. M., Izrailevich Ya. A., Fomenko T. N. Introduction to topology. Moscow, URSS, 2015.

[3] Mukhamadiev E. Bounded solutions and homotopic invariants of systems of nonlinear differential equations. Dokl. Akad. Nauk, 1996, vol. 351, no. 5, pp. 596-598 (in Russian). Doklady Mathematics, 1996, vol. 54. no. 3. pp. $923-925$.

[4] Mukhamadiev E. On the construction of the correct guiding function for a system of differential equations. Trudy Instituta Matematiki Natsional'noy Akad. Nauk Belarusi, 1999, vol. 2, no. 1, pp. 119-127 (in Russian).

[5] Mukhamadiev E., Naimov A. N. Criteria for the existence of periodic and bounded solutions of three-dimensional systems of differential equations. Trudy Instituta Matematiki i Mekhaniki UrO RAN, 2021, vol. 27, no. 1, pp. $157-172$.

DOI: https://doi.org/10.21538/0134-4889-2021-27-1-157-172

Received March 04, 2021.

In revised form, May 13, 2021.

Accepted May 18, 2021.

Published online May 24, 2021. 
E. Mukhamadiev ${ }^{a}$

E-mail: emuhamadiev@rambler.ru

A. N. Naimov ${ }^{a, b}$

E-mail: naimovan@vogu35.ru

a. Vologda State University

15 Lenina st., Vologda 160000, Russia

b. Vologda Institute of Law and Economics of the Federal Penitentiary Service

2 Shchetinina st., Vologda 160002, Russia 